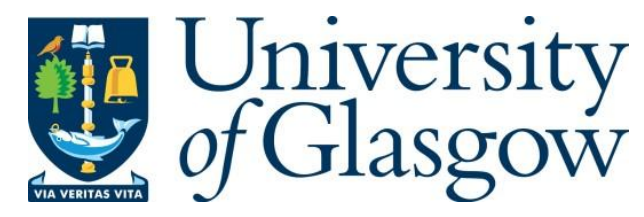

Yang, L., Zare-Behtash, H., Erdem, E., and Kontis, K. (2012) Investigation of the double ramp in hypersonic flow using luminescent measurement systems. Experimental Thermal and Fluid Science, 40 . pp. 50-56. ISSN 0894-1777(doi:10.1016/j.expthermflusci.2012.01.032)

Copyright $\odot 2012$ Elsevier

A copy can be downloaded for personal non-commercial research or study, without prior permission or charge

Content must not be changed in any way or reproduced in any format or medium without the formal permission of the copyright holder(s)

When referring to this work, full bibliographic details must be given

http://eprints.gla.ac.uk/84623/

Deposited on: 07 January 2014

Enlighten - Research publications by members of the University of Glasgow http://eprints.gla.ac.uk 


\title{
Investigation of the Double Ramp in Hypersonic Flow using Luminescent Measurement Systems
}

\author{
L. Yang, H. Zare-Behtash, ${ }^{*}$ E. Erdem, and K. Kontis \\ School of Mechanical, Aerospace and Civil Engineering, University of Manchester, UK
}

\begin{abstract}
Compression ramp flows in supersonic and hypersonic environments present unique flow patterns for shock wave-boundary layer interaction studies. They also represent the generic geometry of two-dimensional inlets and deflected control surfaces for re-entry vehicles. Therefore, a detailed knowledge of the flow behaviour created by such geometries is critical for optimum design. The flow is made more complicated due to the presence of separation regions and streamwise Görtler vortices. The objective of the current research is to study the behaviour and characteristics of the flow over the double ramp model placed in hypersonic flow at freestream Mach number of 5 . Three different incidence angles of $0,-2$, and -4 degrees are studied using colour Schlieren and luminescent paints consisting of anodized aluminium pressure-sensitive paint (AA-PSP) and the temperaturesensitive paint (TSP) technique. The colour Schlieren provides description of the external flow while the global surface pressure and temperature distribution is obtained through the AA-PSP and TSP methods. The TSP technique also proves that it is very effective in identifying the location and properties of the Görtler vortices; revealing the effect of incidence on the magnitude and pattern of Görtler vortices formed.
\end{abstract}

Keywords: Flow Visualisation, Anodized-Aluminium Pressure Sensitive Paint, Temperature Sensitive Paint, Görtler Vortices

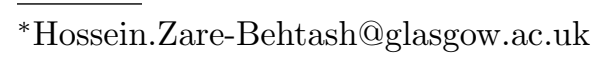




\section{INTRODUCTION}

Supersonic and hypersonic flows over compression ramps present a unique opportunity to study: shock waves, shock-shock interactions, boundary layer instabilities, shock-boundary layer interaction, flow separation and reattachment, and streamwise vortices. ${ }^{1,2}$ The compression ramp also represents a generic geometry such as a deflected control surface or even a two dimensional hypersonic intake, making the study of such complicated flows crucial for hypersonic vehicle design. ${ }^{3,4}$ In most research work, the flow pattern is visualised by optical techniques such as Schlieren or shadowgraph. The quantitative data relies on the discreet pressure tap and thermo sensors. These conventional experimental techniques have their intrinsic drawbacks which makes it difficult to distinguish complicated flow phenomenon.

In recent years surface non-intrusive temperature measurement techniques such as infrared (IR) thermography, ${ }^{5-7}$ thermographic phosphors, ${ }^{8,9}$ liquid crystals, ${ }^{10,11}$ and luminescent temperature sensitive paints (TSP), ${ }^{12}$ have been developed and applied. IR thermography, although successfully used in the supersonic/hypersonic wind tunnel testing, requires the expensive IR camera along with special Germanium or Zinc selenide windows. Additionally, the measurement or estimation of surface emissivity and transmittance of the windows and air require exact knowledge. Liquid crystals on the other hand have a narrow measurement range. Temperature sensitive paints and thermographic phosphors both operate using a similar principle based on a photochemical mechanism. Thermographic phosphors usually work at higher temperatures whereas TSP has a range of -196 to $200{ }^{\circ} \mathrm{C} .{ }^{13}$

Visualisation of roughness induced vortices using a ruthenium based TSP was conducted by Matsumura et al. ${ }^{14}$ in a Mach 4 Ludwieg tube. Due to the high spatial resolution and sensitivity of TSP, weak induced streamwise vortices were detected on an axisymmetric scramjet inlet model. Ishiguro et al. ${ }^{15}$ studied the compression corner flow at Mach 10 using TSP. Görtler vortices which appear as striation like structures were observed on the rear of the reattachment line off the heat flux images. These vortices alter the heat transfer coefficient distribution on the wall and under certain conditions cause boundary layer transition, ${ }^{16-18}$ making the measurement of the heat transfer coefficient a critical parameter.

Pressure sensors are the common pressure measurement equipment in the wind tunnel testing. Depending on the size of the model, multiple pressure tappings and transducers might be necessary, leading to large amounts of tubing and wiring. Thus the process is 
time/space consuming and expensive. Tappings must also be limited in number, so the shape of the model and therefore the local pressure distribution is not altered.

The pressure sensitive paint (PSP) method, which is also a luminescent measurement technique, provides a global non-intrusive map of the model surface pressure. ${ }^{13}$ Taghavi et $a l .{ }^{19,20}$ used PSP to obtain pressure data for a multi-jet supersonic ejector. The PSP method was able to successfully capture key flow features such as bubbles of separated flow and the shock cells. Huang et al. ${ }^{21}$ applied PSP on a micro scale to study micro shock structures. Compared to ordinary PSP, the anodized aluminium pressure sensitive paint (AA-PSP) is believed to have a faster response characteristic and high pressure sensitivity. ${ }^{22}$ This is owed to the porous aluminium structure of the model surface obtained through electro-chemical processes.

The current study investigates the flow properties over a double ramp model using luminescent coatings in a hypersonic flow of $M=5$. Different incidence angles of $0,-2$, and -4 degrees are examined. Schlieren visualisation is employed to visualise the external flow pattern. Anodized aluminium pressure sensitive paint and the temperature sensitive paint techniques are utilised to analyse the pressure distribution and heat transfer rate on the double ramp surface.

\section{EXPERIMENTAL SETUP}

\section{A. Hypersonic wind tunnel}

The facility used in the present study is an intermediate blow-down type hypersonic wind tunnel, which uses dry air as the working fluid. The tunnel has a stable run time of 7.5 seconds. The entire system mainly contains a high pressure vessel, vacuum tank, electric heater, axisymmetric nozzle, working section and auxiliary system such as pumping, pressure supply and water cooling system. In the present research, a 152mm diameter Mach 5 axisymmetric nozzle is employed. The gas temperature can be raised from $375 \mathrm{~K}$ to 700 $\mathrm{K}$ to avoid liquefaction in the test section. Meanwhile, the stagnation pressure is range from 6-8 bar. Unit Reynolds number of 4.5 to $15 \times 10^{6} \mathrm{~m}^{-1}$ can be obtained from the aforementioned stagnation pressure and temperature setting.

The tunnel test section is a free-jet type with dimensions $325 \times 325 \times 900 \mathrm{~mm}$ 
(height $\times$ width $\times$ length) having two circular quartz windows of $195 \mathrm{~mm}$ diameter for optical access. The test section is equipped with a three component balance system which includes the measurement sting and supporting arc. The arc allows the angle of attack of the model to be varied in the range of -20 to 20 degrees relative to the freestream direction. Figure 1 shows the double ramp model installed inside the tunnel test section. The variation in flow Mach number and Reynolds number for different runs of the tunnel are $\pm 0.4 \%$ and $\pm 3.7-3.9 \%$, respectively. More detailed description about the wind tunnel was reported by Erdem and Kontis. ${ }^{23}$

The dimensions of the aluminium double ramp model examined are shown in Figure 2. The first ramp angle is 12 degree and second ramp of 22 degree relative to the axis. A 40 $\mathrm{mm}$ long flat shoulder follows the second ramp. The entire model is made of aluminium alloy 6061 chosen for its anodization capabilities. Experimental conditions of total pressure and temperature are monitored using the stagnation pitot and K-type thermocouple probe. The pitot probe is connected to a Kulite pressure transducer (XTE-190M, 0-100psi) and data is acquired by National Instruments (NI) system and operated using Labview. Eight pressure taps are placed along the centreline of double ramp model and surface pressure is measured by Kulite transducers (XTE-190M, 0-70 kPa). Specifications of the experimental conditions are shown in Table I.

TABLE I: Experimental conditions

\begin{tabular}{lr}
\hline Total pressure $(\mathrm{kPa})$ & 645.90 \\
Total temperature $(\mathrm{K})$ & 372.3 \\
Mach number & 5.0 \\
Freestream pressure $(\mathrm{kPa})$ & 1.22 \\
Freestream temperature $(\mathrm{K})$ & 62.5 \\
Unit Reynolds number $\left(\mathrm{m}^{-1}\right)$ & $13.5 \times 10^{6}$ \\
Incidence (degrees) & $0,-2$ and -4 \\
\hline
\end{tabular}

\section{B. Colour Schlieren}

Schlieren allows for the visualisation of invisible light refractions. ${ }^{24,25}$ The benefit of colour Schlieren, used here, is that with the addition of colour it not only becomes easier to discern 
flow features but the intensity of the colour also gives an indication of the magnitude of the flow features.

A z-type Schlieren system similar to that of Erdem et. al. ${ }^{26}$ is employed to visualise the flow. The setup consists of a Palflash 501 continuous light source and two 8 inch diameter parabolic mirrors with a $6 \mathrm{ft}$ focal length. Colour images are acquired by placing a horizontal slit at the source and a 3-colour (red, green, and blue) colour filter at the knife edge location, see Figure 3. A digital Canon SLR camera, EOS-450D, with 12MP resolution is used to capture the images. The camera is set to continuous shooting mode at 3.5 frames per second, while the shutter speed is set to the minimum value of $1 / 4000$ seconds.

\section{AA-PSP}

The PSP technique has become an invaluable tool in aerodynamics, where the emitted intensity of the paint is related to surface pressure. Detailed descriptions of this technique are abundant in literature. ${ }^{13,27-32}$ Anodized Aluminium Pressure Sensitive Paint (AA-PSP) is a relatively new branch of PSP which has a fast response characteristic, enabling the measurement of unsteady phenomena. ${ }^{33,34}$ This is due to the electro-chemical process which AA-PSP uses to create a porous surface on the model to deposit the PSP molecules.

The preparation of the AA-PSP model is similar to the procedure suggested by Sakaue ${ }^{35}$ and Kameda et al. ${ }^{36}$ with a small modification. More details of the model preparation is reported by Yang et al. ${ }^{37}$ The anodized model is then dipped in tris-(Bathophnanthroline) Ruthenium (II) perchloride solution with a concentration of $0.3 \mathrm{mmol} / \mathrm{L}$ with respect to the Dichloromethane solvent volume. Yang et al. ${ }^{38}$ have successfully applied the described AA-PSP method to several models in hypersonic flow.

A pair of light emitting diode (LED) panels with peak wavelength of 470nm are used for illumination. Filters were needed to separate the emitted light from the luminscent paints from excitation light. The spectral analysis of the AA-PSP and TSP is presented in Figure 4. Based on this graph, the blue LEDs which were used for illuminating the AA-PSP are also utilised to excite the TSP. A combination of two filters was used to capture the emitted light from the AA-PSP. The first, an orange long pass filter, allowing the transmission of light with $\lambda>580 \mathrm{~nm}$, and the second filter was an IR cut-off filter, preventing the transmission of light above $\lambda>700 \mathrm{~nm}$. In the meanwhile, the emission from TSP was recorded using long 
pass filter with $\lambda>550 \mathrm{~nm}$ and an IR cut-off filter similar to the AA-PSP. The images were captured using a 12-bit LaVision Image Intense CCD camera. The present camera covers the spectral range from $290 \mathrm{~nm}$ to $1100 \mathrm{~nm}$ but has its maximum quantum efficiency about $65 \%$ at $500 \mathrm{~nm}$. The lowest emission signal is found to be 921 counts $(22.5 \%$ of the full 12-bit camera chip capacity) for the PSP test, which satisfies for the present measurement requirements. The PSP image is taken at 9 fps frame rate with $8 \mathrm{~ms}$ exposure time while TSP is recorded with slightly longer exposure time, $10 \mathrm{~ms}$. A total of 30 images were summed and taken average for processing in order to reduce the noise of the PSP results. Since the surface temperature of model keeps rising during the test. Such method can not be put in to the TSP data processing. Only single image was used for calculation of TSP data which, unfortunately, leads to a noisy signal.

Essentially, temperature dependency has always been an inherent characteristic of PSP due to the thermal quenching mechanism. The temperature sensitivity of the current AAPSP formulation is found to be approximately $-0.64 \%$ per degree. ${ }^{39}$ The temperature rise during the test duration will cause considerable error if it is uncorrected. However, in the current setup in-situ calibration was applied to convert the AA-PSP images to pressure maps. The advantage of in-situ calibration is that it can absorb the temperature effect to the fitting error thus eliminates the error caused by temperature dependency. ${ }^{40}$ This was done by taking pressure measurements along the double ramp surface during a run and relating the pressure values to the intensity of the AA-PSP in the immediate vicinity. Figure 5 is a plot of intensity ratio vs pressure ratio, known as a Stern-Volmer graph, the points refer to the transducer measurements. Both pressure and intensity are non-dimensionalised with respect to a reference condition. The coefficients of the line of best fit are then used to calibrate the AA-PSP images.

\section{Temperature Sensitive Paint (TSP)}

Besides visualisation, TSP can also provide accurate quantitative measurement of surface temperature. Nagai et $a .^{41}$ examined the effect of dye concentration on the signal level and temperature sensitivity of TSP which used $\mathrm{Ru}(\text { phen })_{3}^{2+}$ as the dye molecules. It was concluded that $10^{-2} \mathrm{~mol} / \mathrm{L}$ of dye is the most suitable concentration for the recipe used. The composition of TSP used in the current research is similar to the recipe presented by Ishiguro 
et $a l .{ }^{15}$ and Nagai et al. ${ }^{41} 71.2 \mathrm{mg}$ of tris-(1,10-phenanthroline) ruthenium (II) chloride is diluted in $10 \mathrm{ml}$ of polyacrylic acid, which corresponds to a concentration of $10^{-2} \mathrm{~mol} / \mathrm{L}$. Afterwards, the mixture is diluted with $20 \mathrm{ml}$ of ethanol and put in an ultrasonic bath to mix. Here, ethanol works as the solvent and polyacylic acid forms a gas-impermeable layer on the model to minimise the pressure sensitivity of the TSP.

A $20 \times 20 \mathrm{~mm}$ TSP sample was calibrated using a static calibration rig where the temperature and pressure are digitally controllable. The coupon was coated with exactly the same TSP solution and spraying thickness as the one used for wind tunnel testing. The relationship between intensity and temperature is provided in Figure 6. Similar to the AA-PSP calibration, the coefficients of the line of best fit are applied to the TSP images acquired during a tunnel run to obtain temperature maps.

The pressure sensitivity of TSP is also an important parameter that determines whether a specific formula is purely temperature sensitive or exhibits a level of pressure sensitivity as well which is not desirable. Figure 7 shows the variation of intensity with pressure at a constant temperature. The current TSP formulation shows a pressure sensitivity of $0.15 \%$ per bar. The photodegredation of the TSP was also examined in Figure 8. The paint displays a decrease in intensity of $0.0011 \%$ per minute.

When applying TSP to the double ramp model, since the model is made of aluminium, a $75 \mu \mathrm{m}$ Mylar film was utilised as a thermal insulating layer and glued on to the model surface. This approach was also adopted by Ishiguro et al. ${ }^{15}$ It is believed that this insulating layer will prevent heat dissipation so that temperature signal level of TSP can be increased and also the signal noisy would be minimize. The TSP solution was sprayed on the sample with 12 layers. An adhesive type of K-type thermocouple was attached to the under side of the model to record the initial pre-test temperature.

Before each test, a wind-off reference image was recorded at a known temperature. A dark image was also taken and subtracted from the raw reference and testing images for dark noise compensation.

\section{RESULTS AND DISCUSSIONS}

Figure 9 shows the colour Schlieren for an angle of attack of 0 degrees and AA-PSP contours for the double ramp at incidences of 0 and -4 degrees. The filled circles in the 
AA-PSP correspond to pressure taps used for AA-PSP in-situ calibration and pressure measurement validation. The first dashed line on the AA-PSP result indicates the hinge between the first and second ramp whilst the second dashed line represents the starting of the shoulder section of the model. The leading edge shock, weak separation shock, reattachment shock, separation region, and the thicker boundary layer in the rear of the reattachment line are identifiable from the Schlieren image. The shock wave angle and strength relates to the ramp angle and freestream Mach number. A separation region is formed around the corner junction of the first and second ramp due to the adverse pressure gradient imposed by the second ramp surface. A weak separation shock wave is created in front of the separation region. A "necking" region of boundary layer can be seen on the second ramp from Schlieren, which indicates the boundary layer re-establishment after flow re-attachment. The blue colour of the Schlieren image on the second ramp also clearly shows the thicker boundary layer, this might suggest boundary layer transition due to flow re-attachment. A PrandtlMeyer expansion fan forms on the shoulder to guide the flow parallel to the model surface whilst accelerating it.

The AA-PSP result captures the main flow pattern. The highest pressure visible on the PSP results occurs on the second ramp since the incoming flow is compressed by both the leading edge and re-attachment shock waves. A three dimensional effect is observed on the AA-PSP contour, which is mostly evident on the first ramp and flat shoulder. Additionally, the contours show the increase in pressure on the first and second ramp surfaces as the incidence reduces.

The normalised pressure profile from the AA-PSP results along the model centreline is plotted in Figure 10 at the different incidences along with Kulite transducer data and inviscid theoretical prediction. The theoretical pressure ratio is calculated from oblique shock wave and Prandtl-Mayer expansion wave equations. The maximum absolute noise level of the AAPSP signal was obtained at the location of the second ramp as 262.3 Pa which corresponds to just $2.57 \%$ of the averaged absolute pressure value. A good agreement can be seen between the AA-PSP, theoretical prediction and Kulite transducer data with the exception of second ramp surface. For all the angles studied the AA-PSP measurement shows a maximum of $9 \%$ difference in comparison to the Kulite pressure transducer data. The discrepancy between the theory and AA-PSP on the second ramp is attributed to the viscous effect. A zoom-in plot of the corner junction is also shown for clarification of the separation region. As the 
incidence is reduced, the effective wedge angle of the ramp increases, requiring a stronger shock to guide the flow parallel to the model surface. Therefore, the strength of the leading edge shock increases with decreasing incidence (from 0 to -4 degree) with a decrease in Mach number behind the shock. This leads to a weaker re-attachment shock created at the lower incidence. The size of separation region is found slightly samller at the lower incidence as well. The separation length, which is measured from the Schlieren images with $\pm 0.1 \mathrm{~mm}$ uncertainty ,reduces from $8.98 \mathrm{~mm}$ at 0 degrees incidence to $7.99 \mathrm{~mm}$ at -4 degrees incidence.

The heat-flux rate was obtained by integrating the surface temperature history based on a 1-D heat conduction assumption. Here, an approximated discretised equation is adapted based on the work of Cook and Felderman, ${ }^{42}$

$$
q(t)=\frac{2 \sqrt{\rho c k}}{\sqrt{\pi}}\left[\sum_{i=1}^{n} \frac{T\left(t_{i}\right)-T\left(t_{i-1}\right)}{\sqrt{t_{n}-t_{i}}+\sqrt{t_{n}-t_{i-1}}}\right]
$$

where $\rho$ is the density, $c$ is the heat capacity, and $k$ is the thermal conductivity. More details can be found from Schultz and Jones. ${ }^{43}$ For a calorically perfect gas, the heat flux can be represented as a non-dimensional parameter known as the Stanton number, $C_{H}:{ }^{44}$

$$
C_{H}=\frac{q}{c_{p} \rho_{e} u_{e}\left(T_{a w}-T_{w}\right)}
$$

where $\rho_{e}$ and $u_{e}$ are the density and velocity at the boundary layer edge which are taken to be equal to the freestream values, $T_{a w}$ and $T_{w}$ are the adiabatic wall temperature and wall temperature, respectively. $T_{a w}$ is calculated using Eqs. (3) and (4). For air, the Prandtl number $(\mathrm{Pr})$ can be assumed as 0.715 .

$$
\begin{gathered}
T_{a w}=\gamma\left(T_{o}-T_{e}\right)+T_{e} \\
\gamma=\sqrt{P r}
\end{gathered}
$$

The surface temperature history is acquired through the TSP images. By integrating the surface temperature history according to Eq.(1) the heat flux on the model surface is obtained. The streamwise heat flux distribution along the centreline of the model at all incidences tested is shown in Figure 11. Due to the limited field of view, only a portion of the entire model can be seen. Unfortunately, a very smooth heat flux profile has not been obtained. The reason for this is mainly attributed to the following reasons: firstly, with the viscous TSP solution, it is very difficult to achieve a fine mist using an airbrush. This 
causes a certain level of noise in the time history of the temperature profile. Furthermore, the equation for heat flux calculation amplifies the levels of noise from the temperature reading. These all lead to the relatively noisy heat flux profile. However, the overall trend of heat flux distribution is still well captured. At the current experimental conditions, the unit Reynolds number is $13.5 \times 10^{6} \mathrm{~m}^{-1}$ and the flow is believed to be laminar based on the local Reynolds number. The heat flux gradually declines along the first ramp as the boundary layer develops before encountering the second ramp surface. A sudden decrease of heat flux can be seen from the profile before the corner between the first and second ramps at around $\mathrm{x} / \mathrm{L}=0.4$, which indicates the start of the separation zone. In general, the separation regions in high speed turbulent flows result in increased heat transfer whereas for laminar separated regions show a reduction in heat transfer. ${ }^{45}$ Therefore it is another proof of the laminar separation.

Immediately downstream of the separation region, the heat flux peak corresponds to the flow reattachment, where there is lcoal thinning of boundary layer thickness so called "necking region" as shown in Figure 9 Schlieren image. The reattachment and the associated necking region can cause transition to occur downstream where flow regains momentum after reattachment. ${ }^{5}$ This plateau clearly indicates the transition mechanism as heat flux does not diminish with redeveloping/growing boundary layer. The transition is also suggested in the colour Schlieren image in Figure 9. Further downstream, the heat flux reduces as a result of the expansion fan present at the apex of the corner.

As the model incidence reduces, the heat flux lifts up which is more apparently on the first ramp surface. This is mainly caused by the increased strength of leading edge shock wave. The increased heat flux also appears on the second ramp surface at lower incidence.

The temperature map on the second ramp surface is presented in Figure 12. Large variations of spanwise temperature occur on the model. The striations on the ramp surface, indicate the existence of Görtler vortices. When flow passes a curved surface, Görtler vortices are generated due to the centrifugal force. Pairs of counter-rotating vortices, starting from the boundary layer reattachment line, lift up from model surface and develop to downstream. A schematic of the vortices structure is shown in Figure 13. Where two adjacent vortices impinge on the model surface high temperature strips are created, at the same time, where two adjacent vortices depart from the surface, low temperature areas are formed. Higher temperature steaks occur at lower incidence. A three-dimensional effect is 
also visible on the second ramp, where the vortices are inclined to the model sides when developing downstream because of spillage. The existence of spanwise heating striation on the second ramp is in agreement with the infra-red thermography measurement on compression ramp in Mach 6 flow by Simeonides. ${ }^{5}$ The Görtler vortices are found with relation to the laminar-turbulent transition after flow reattachment, which has been demonstrated by a number of investigations. ${ }^{46-48}$ Thesse high temperature striations may cause severe heating problem on the model surface.

Profiles of spanwise Stanton number for an incidence angle of -4 are provided in Figure 14. The oscillation of the Stanton number further confirms the existence of Görtler vortices. The average value of spanwise Stanton number, represented by the solid line, shows an increase in Stanton number with downstream distance on the second ramp surface. This behaviour indicates the unstable of Görtler vortices development. For all the cases studied, an increased Stanton number is observed with downstream distance. The Görtler vortices develop further with downstream distance and lift up from the ramp surface. At the lower the incidences, the heat transfer is more severe.

\section{CONCLUSIONS}

The flow features of a double ramp model in hypersonic flow, at different incidences, have been visualised and their properties quantified using luminescent measurements of temperature and pressure. To better understand the flow field, the TSP technique along with colour Schlieren and AA-PSP methods are employed. Complex flow patterns including the separation region, flow reattachment, and three dimensional effects were captured in high resolution from the measurements, correlating well with the flow physics expected from such flows. The results show that the separation region reduces as the incidence is lowered from 0 to -4 degrees. Qualitatively, AA-PSP captures the main structure of flow field which closely matches that visualised by colour Schlieren. The pressure measured by the AA-PSP shows a maximum of $9 \%$ difference with the data acquired from Kulite pressure transducers.

Other than the surface pressure, heat flux was also obtained by integrating surface temperature history measured using the TSP technique. Compared to the 0 degrees incidence,

the heat flux was found to increase at lower incidences. Striations were present on the second ramp surface with large variations of Stanton number in the spanwise direction. However, 
the TSP was not smooth enough to provide a low noise signal when examining the surface profile. The future work is concentrated on obtaining a smooth TSP paint surface and further investigating the surface flow pattern.

\section{Acknowledgments}

The authors are indebted to the technical and administrative staff at The University of Manchester for their assistance. The scholarship provided by the UK-China Scholarships for Excellence programme for the first author is highly appreciated. The support of the EPSRC Engineering Instrument Pool for the loan of the spectrometer and laser surface profiler are greatly acknowledged as well.

1 Zheltovodov, A.A., " Shock waves/turbulent boundary-layer interactions - Fundamental studies and applications," Fluid Dynamics Conference, 27th, New Orleans, AIAA-1996-1977, 1996.

2 Loginov, M.S., Adams, N.A., Zheltovodov, A.A., "Large-eddy simulation of shockwave/turbulent-boundary-layer interaction," Journal of Fluid Mechanics 565, pp. 135-169, 2006.

3 Berry, S.C., Auslender, A.H., Dilley, A.D., Calleja, J.F., "Hypersonic boundary-layer trip development for Hyper-X," Journal of Spacecraft and Rockets 38, pp. 853-864, 2001.

4 Schrijer, F.F.J., Scarano, F., van Oudheusden, B.W., "Application of PIV in a Mach 7 doubleramp flow," Experiments in Fluids 41, pp. 353-363, 2006.

5 Simeonides, G., Haase, W., "Experimental and computational investigations of hypersonic flow about compression ramps," Journal of Fluid Mechanics 283, pp. 17-42, 1995.

6 Aymer de la Chevalerie, D., Fonteneau, A., De Luca, L., Cardone, G., "Görtler-type vortices in hypersonic flows: the ramp problem," Experimental Thermal and Fluid Science 15, pp. 69-81, 1997.

7 Marini, M., "Analysis of hypersonic compression ramp laminar flows under sharp leading edge conditions," Aerospace Science and Technology 5, pp. 257-271, 2001.

8 Khalid, A., Kontis, K., "Thermographic phosphors for high temperature measurements: principles, current state of the art and recent applications," Sensors 8, pp. 5673-5744, 2008. 
9 Khalid, A., Kontis, K., Behtash, H.Z., "Phosphor thermometry in gas turbines: consideration factors ," Proceedings of the Institution of Mechanical Engineers, Part G: Journal of Aerospace Engineering 224, pp. 745-755, 2010.

10 Roberts, G.T., East, R.A., "Liquid crystal thermography for heat transfer measurement in hypersonic flows - A review," Journal of Spacecraft and Rockets 33, pp. 761-768, 1996.

11 Babinsky, H., Edwards, J.A., "Automatic liquid crystal thermography for transient heat transfer measurements in hypersonic flow," Experiments in Fluids 21, pp. 227-236, 1996.

12 Liu, T., Campbell, B.T., Sullivan, J.P., "Fluorescent paint measurement of heat transfer in shock - turbulent boundary layer interaction," Experimental Thermal and Fluid Science 10, pp. 101-112, 1995.

13 Liu, T., Sullivan, J.P., "Pressure and temperature sensitive paints," Springer Verlag, 2005.

14 Matsumura, S., Huang, C.-Y., Choi, Y.-S., Swanson, E.O., Salyer, T.R., Sakaue, H, "Feasibility of detecting streamwise vortices from roughness elements using temperature sensitive paint in a Mach 4 Ludweig tube," 22nd AIAA Aerodynamic Measurement Technology and Ground Testing Conference, St. Louis, AIAA-2002-3238, 2002.

15 Ishiguro, Y., Nagai, H., Asai, K., Nakakita, K., "Visualization of hypersonic compression corner flows using temperature- and pressure-sensitive paints," 45th AIAA Aerospace Sciences Meeting and Exhibit, Reno, AIAA-2007-118, 2007.

16 Luca, L., Cardone, G., Aymer de la Chevalerie, D., Fonteneau, A., "Gortler instability of a hypersonic boundary layer," Experiments in Fluids 16, pp. 10-16, 1993.

17 Saric, W.S., "Görtler vortices," Annual Review of Fluid Mechanics 26, pp. 379-409, 1994.

18 Winoto, S.H., Mitsudharmadi, H., Shah, D.A., "Visualizing Görtler vortices," Journal of Visualization 8, pp. 315-322, 2005.

19 Taghavi, R., Raman, G., Bencic, T.J., "Pressure sensitive paint demonstrates relationship between ejector wall pressure and aerodynamic performance," Experiments in Fluids 26, pp. 481-487, 1999.

20 Taghavi, R., Raman, G., Bencic, T.J., "Mixer-ejector wall pressure and temperature measurements based on photoluminescence," AIAA Journal 40, pp. 745-750, 2002.

21 Huang, C.Y., Gregory, J.W., Sullivan, J.P., "Flow visualization and pressure measurement in micronozzles," Journal of Visualization 10, pp. 123-130, 2007.

${ }^{22}$ Gregory, J.W., Asai, K., Kameda, M., Liu, T., Sullivan, J.P., "A review of pressure-sensitive 
paint for high-speed and unsteady aerodynamics," Proceedings of the Institute of Mechanical Engineers, Part G: Journal of Aerospace Engineering 222, pp. 249-290, 2008.

23 Erdem, E., Kontis, K., "Non-plasma and plasma transverse jets in hypersonic cross flow," 16th AIAA/DLR/DGLR International Space Planes and Hypersonic Systems and Technologies Conference, Germany, AIAA-2009-7253, 2009.

24 Settles, G.S., "Colour-coding schlieren techniques for the optical study of heat and fluid flow," International Journal of Heat and Fluid Flow 6, pp. 3-15, 1985.

25 Settles, G.S., "Schlieren and shadowgraph techniques," Springer Verlag, 2001.

26 Erdem, E., Yang, L., Kontis, K., "Drag reduction by energy deposition in hypersonic flows," 16th AIAA/DLR/DGLR International Space Planes and Hypersonic Systems and Technologies Conference, Germany, AIAA-2009-7347, 2009.

27 Carroll, B.F., Abbitt, J.D., Lucas, E.W., Morris, M.J., "Step Response of Pressure-Sensitive Paints," AIAA Journal 34, pp. 521-526, 1996.

28 Mosharov, V., Radchenko, V., Fonov, S., "Luminescent Pressure Sensors in Aerodynamic Experiments," Central Aerodynamic Institute (TsAGI), 1998.

29 Bell, J.H., Schairer, E.T., Hand, L.A., Mehta, D., "Surface pressure measurements using luminescent coatings," Annual Review of Fluid Mechanics 33, pp. 155-206, 2001.

30 Zare-Behtash, H., Gongora-Orozco, N., Kontis, K., "PSP visualization studies on a convergent nozzle with an ejector system," Journal of Visualization 12, pp. 157-163, 2009.

31 Zare-Behtash, H., Gongora-Orozco, N., Kontis, K., Holder, S.J., "Application of novel pressuresensitive paint formulations for the surface flow mapping of high-speed jets," Experimental Thermal and Fluid Science 33, pp. 852-864, 2009.

32 Basu, B.J., Vasantharajan, N., Raju, C., "A novel pyrene-based binary pressure sensitive paint with low temperature coefficient and improved stability," Sensors and Actuators B: Chemical 138, pp. 283-288, 2009.

33 Sakaue, H., "Porous pressure-sensitive paint for characterizing unsteady flowfields," AIAA Journal 40, pp. 1094-1098, 2002.

34 Liu, T., Teduka, N., Kameda, M., Asai, K., "Diffusion timescale of porous pressure-sensitive paint," AIAA Journal 39, pp. 2400-2402, 2001.

35 Sakaue, H., "Luminohpore application method of anodized aluminium pressure sensitive paint as a fast responding global pressure sensor," Review of Scientific Instruments 76, pp. 1-6, 2005. 
${ }^{36}$ Kameda, M., Tezuka, N., Hangai, T., Asai, K., Nakakita, K., Amao, Y., "Adsorptive pressuresensitive coatings on porous anodized aluminium," Measurement Science and Technology 15, pp. 489-500, 2004.

37 Yang, L., Zare-Behtash, H., Erdem, E., Kontis, K., "Application of AA-PSP to Hypersonic Flows: The Double Ramp Model," Sensors and Actuators B: Chemical In press.

38 Yang, L., Erdem, E., Kontis, K., "Pressure-sensitive paint application on two-dimensional and axisymmetric model in hypersonic flow," 49th AIAA Aerospace Sciences Meeting including the New Horizons Forum and Aerospace Exposition, Orlando, AIAA-2011-849, 2011.

39 Quinn, M.K., Yang, L., Kontis, K., "Pressure-Sensitive Paint: Effect of Substrate," Sensors 11, pp. 11649-11663, 2011.

40 Liu, T., Sullivan, J.P., "In situ calibration uncertainty of pressure-sensitive paint," AIAA Journal 41, pp. 2300-2302, 2003.

41 Nagai, H., Ohmi, S., Asai, K., Nakakita, K., "Effect of temperature-sensitive-paint thickness on global heat transfer measurement in hypersonic flow," Journal of Thermophysics and Heat Transfer 22, pp. 373-381, 2008.

42 Cook, W.J., Felderman, E.J., "Reduction of data from thin-film heat-transfer gages: A concise numerical technique," AIAA 4, pp. 561-562, 1966.

43 Schultz, D.L., Jones, T.V., "Heat-transfer measurements in short-duration hypersonic facilities," AGARDograph, AGARD-AG-165, 1973.

44 Anderson Jr., J.D., "Hypersonic and High-Temperature Gas Dynamics," AIAA Education, 2006.

45 Coleman, G.T., Stollery, J.L., "Heat transfer from hypersonic turbulent flow at a wedge compression corner," Journal of Fluid Mechanics 56, pp. 741-752, 1972.

46 Simeonides, G., "Hypersonic shock wave boundary interactions over compression corners," PhD Thesis, University of Bristol/von Karman Institute, 1992.

47 Arnal, D., "Laminar-turbulent transition problems in supersonic and hypersonic flows," AGARD-FDP/VKI Special Course on Aerothermodynamics of Hypersonic Vehicles, AGARD Report, 1989.

48 Floryan, J.M., "On the Görtler instability of boundary layers," Progress in Aerospace Sciences 28, pp. 235-271, 1991. 


\section{List of Figures}

1 Test section of the hypersonic wind tunnel with installed model. . . . . . . . 17

2 Dimension of double ramp model. . . . . . . . . . . . . . . . . . . . 17

3 Colour Schlieren light source slit and colour filter. . . . . . . . . . . . . . 17

4 Emission and excitation spectra of AA-PSP and TSP . . . . . . . . . 18

5 In-situ calibration of the AA-PSP . . . . . . . . . . . . . . . 18

6 Temperature sensitivity of $\mathrm{TSP}, \mathrm{T}_{\text {ref }}=21.5^{\circ} \mathrm{C}$ and $\mathrm{P}=101 \mathrm{kPa} . \quad \ldots \ldots$

$7 \quad$ Pressure sensitivity of $\mathrm{TSP}, \mathrm{T}=21.5^{\circ} \mathrm{C}$ and $\mathrm{P}_{\text {ref }}=1.3$ bar. . . . . . . . . . . 19

8 Photodegradation of TSP signal with time. . . . . . . . . . . . . 20

9 Colour Schlieren and AA-PSP contours of the double ramp. . . . . . . . . 21

10 Centreline pressure profile from AA-PSP at difference incidences. . . . . . . 22

11 Variation of heat flux along the model centreline at different incidences. . . . 22

12 TSP of the Görtler vortices formed on the second ramp at different incidences. 23

13 Flow pattern induced by the Görtler vortices adjacent to the model surface. ${ }^{15} 23$

14 Spanwise variation of Stanton number at different locations for an incidence of -4 degrees. . . . . . . . . . . . . . . . . . . . . . . 24 


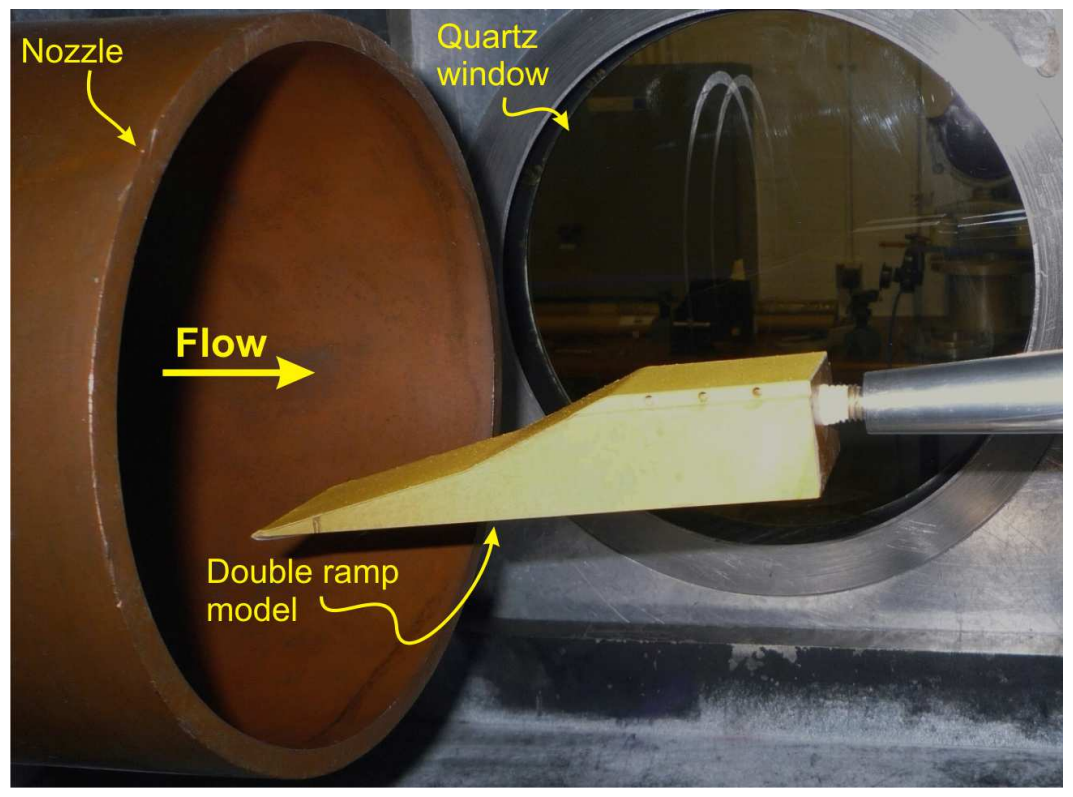

FIG. 1: Test section of the hypersonic wind tunnel with installed model.

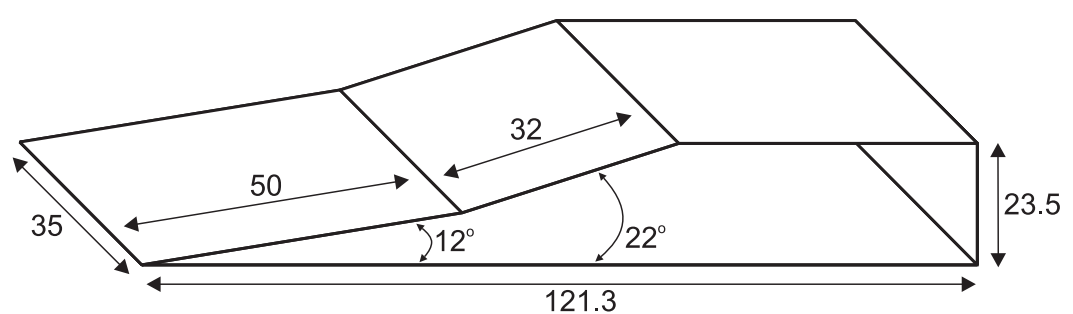

FIG. 2: Dimension of double ramp model.

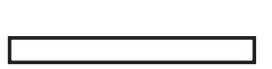

Source

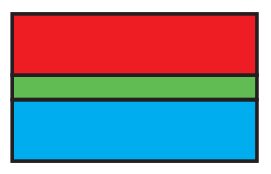

Knife-edge

FIG. 3: Colour Schlieren light source slit and colour filter. 


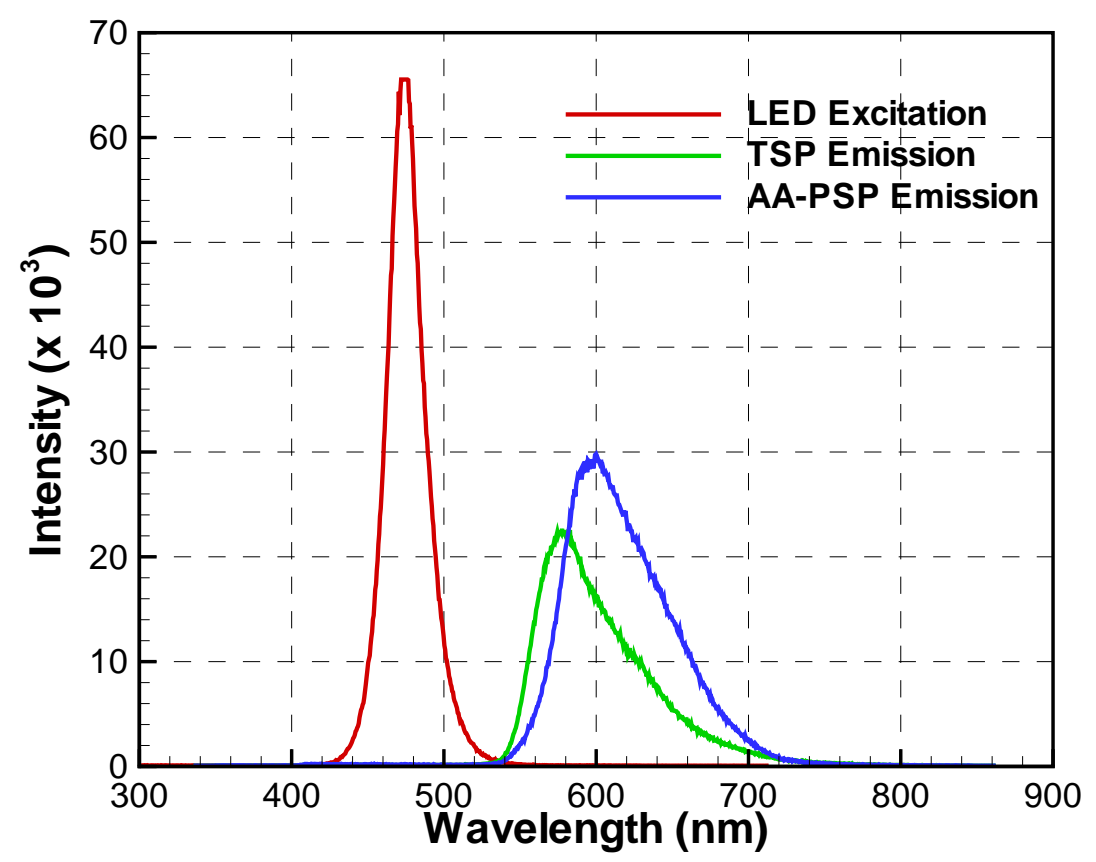

FIG. 4: Emission and excitation spectra of AA-PSP and TSP.

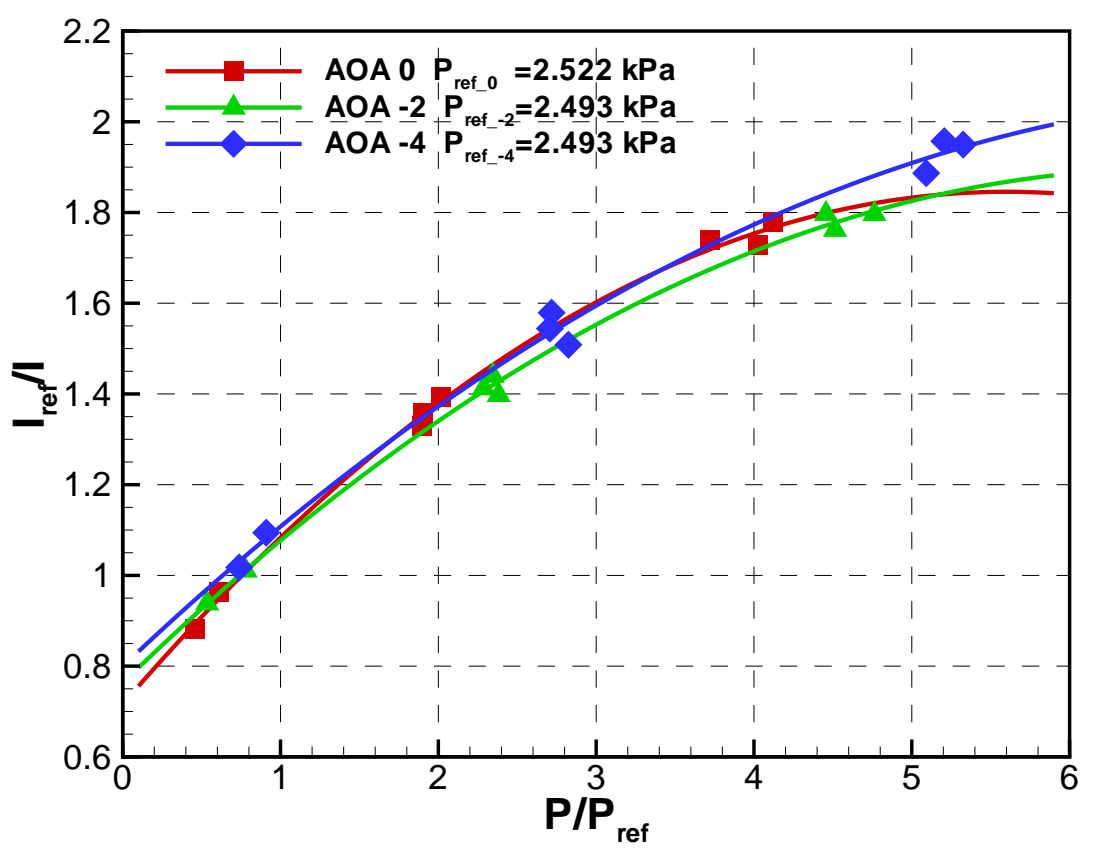

FIG. 5: In-situ calibration of the AA-PSP. 


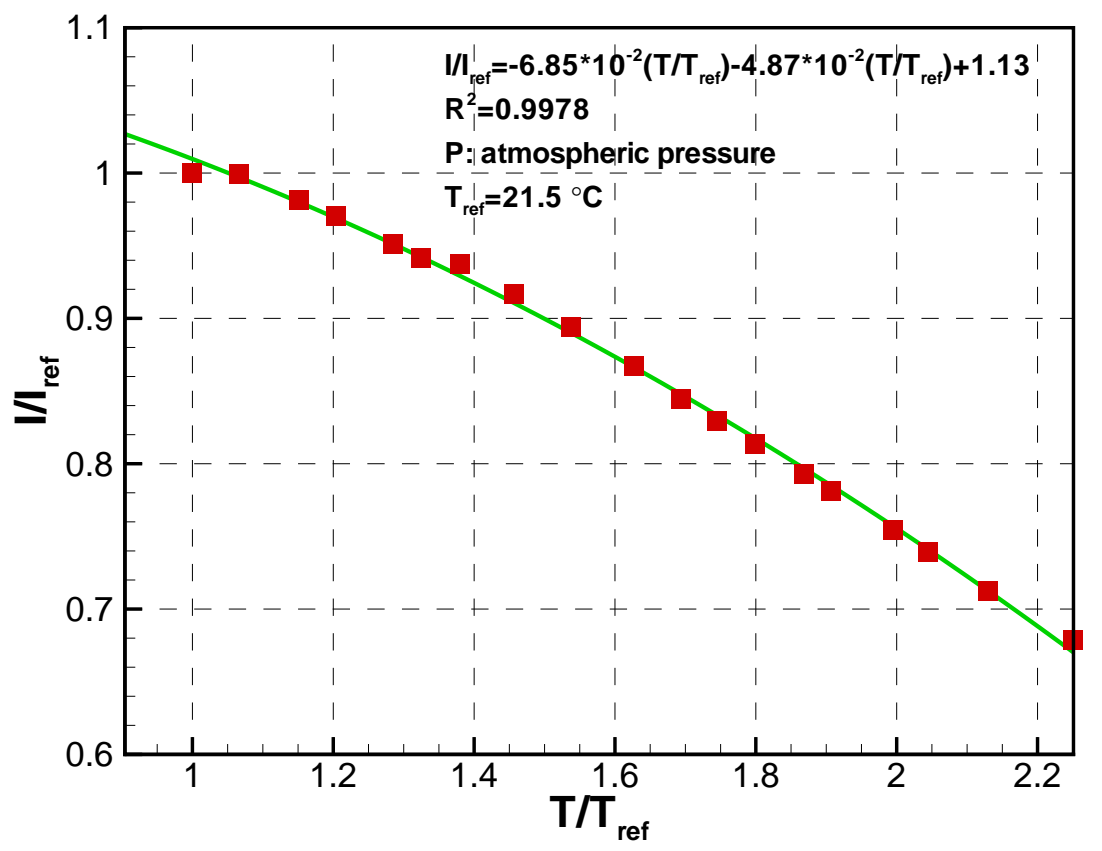

FIG. 6: Temperature sensitivity of TSP, $\mathrm{T}_{\text {ref }}=21.5^{\circ} \mathrm{C}$ and $\mathrm{P}=101 \mathrm{kPa}$.

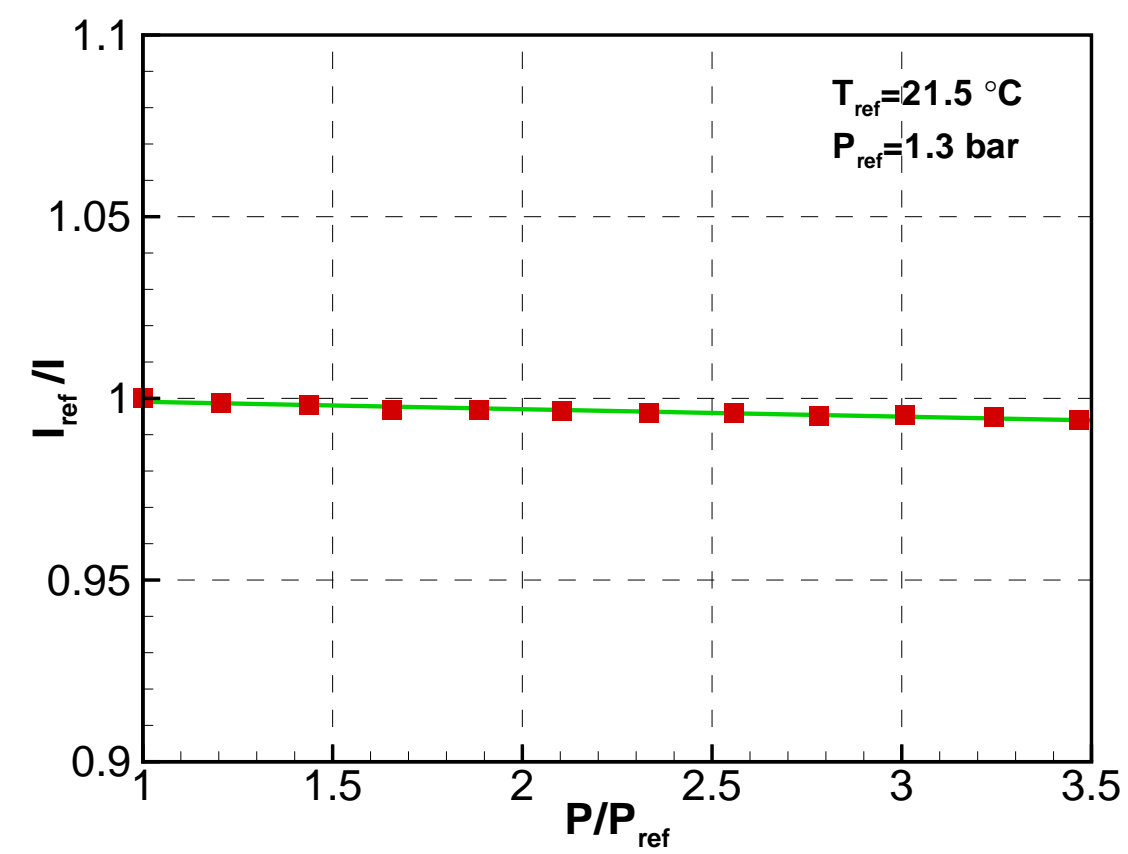

FIG. 7: Pressure sensitivity of TSP, $\mathrm{T}=21.5^{\circ} \mathrm{C}$ and $\mathrm{P}_{\text {ref }}=1.3 \mathrm{bar}$. 


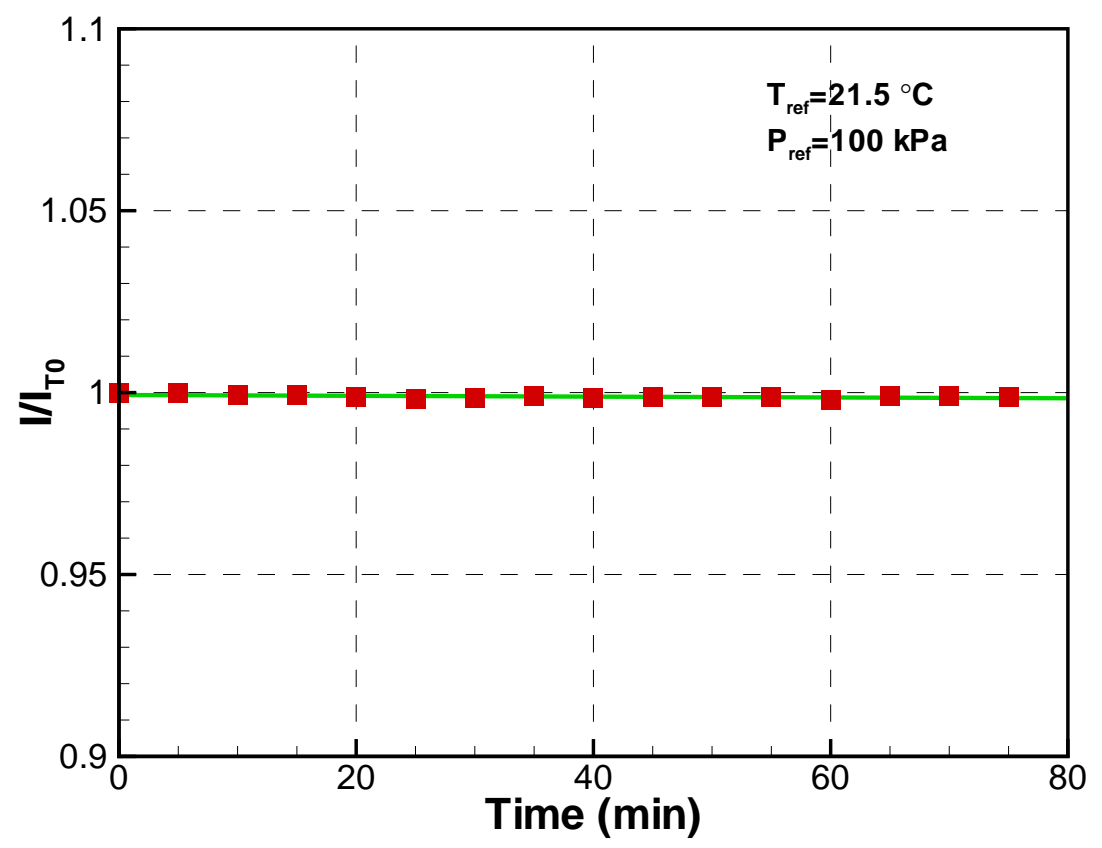

FIG. 8: Photodegradation of TSP signal with time. 


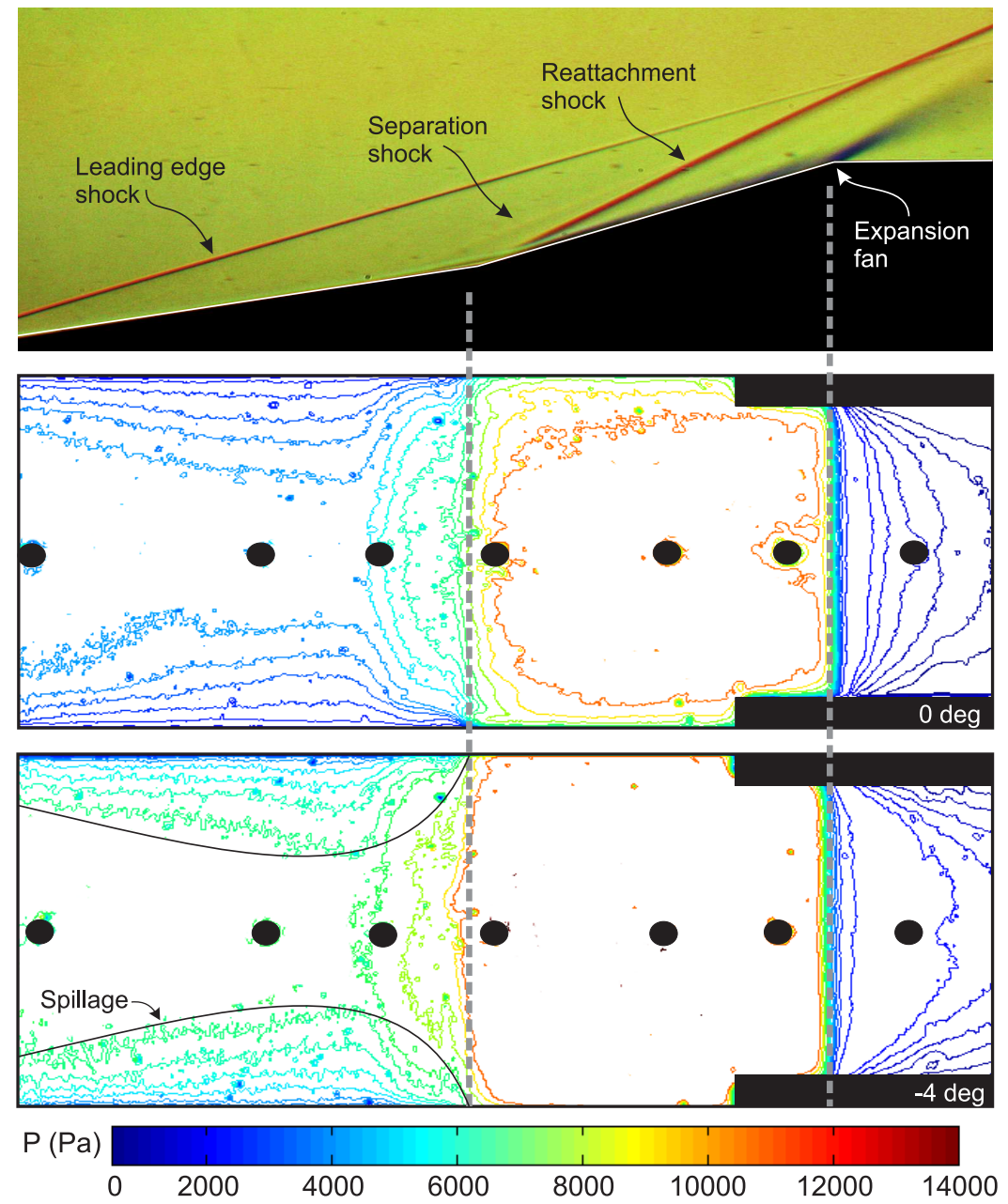

FIG. 9: Colour Schlieren and AA-PSP contours of the double ramp. 


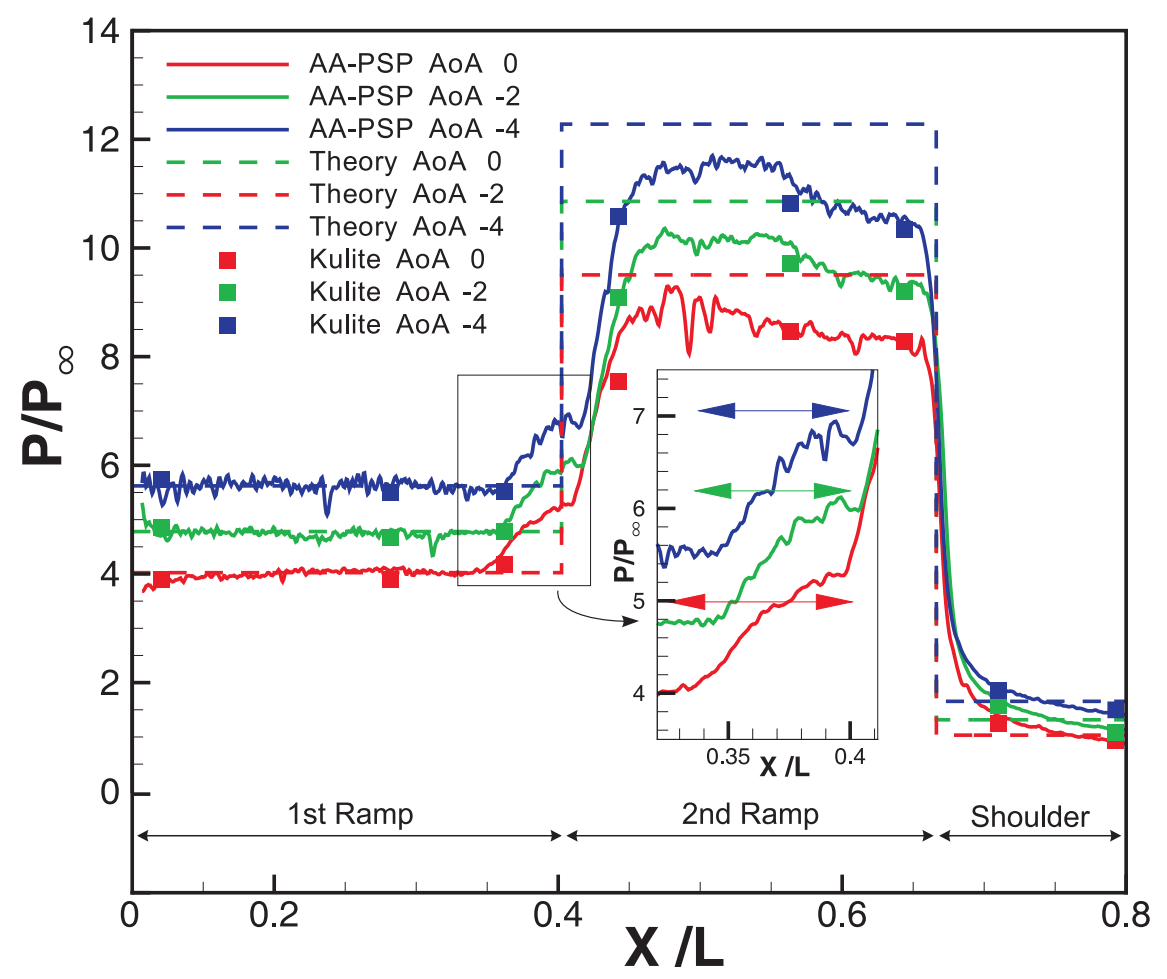

FIG. 10: Centreline pressure profile from AA-PSP at difference incidences.

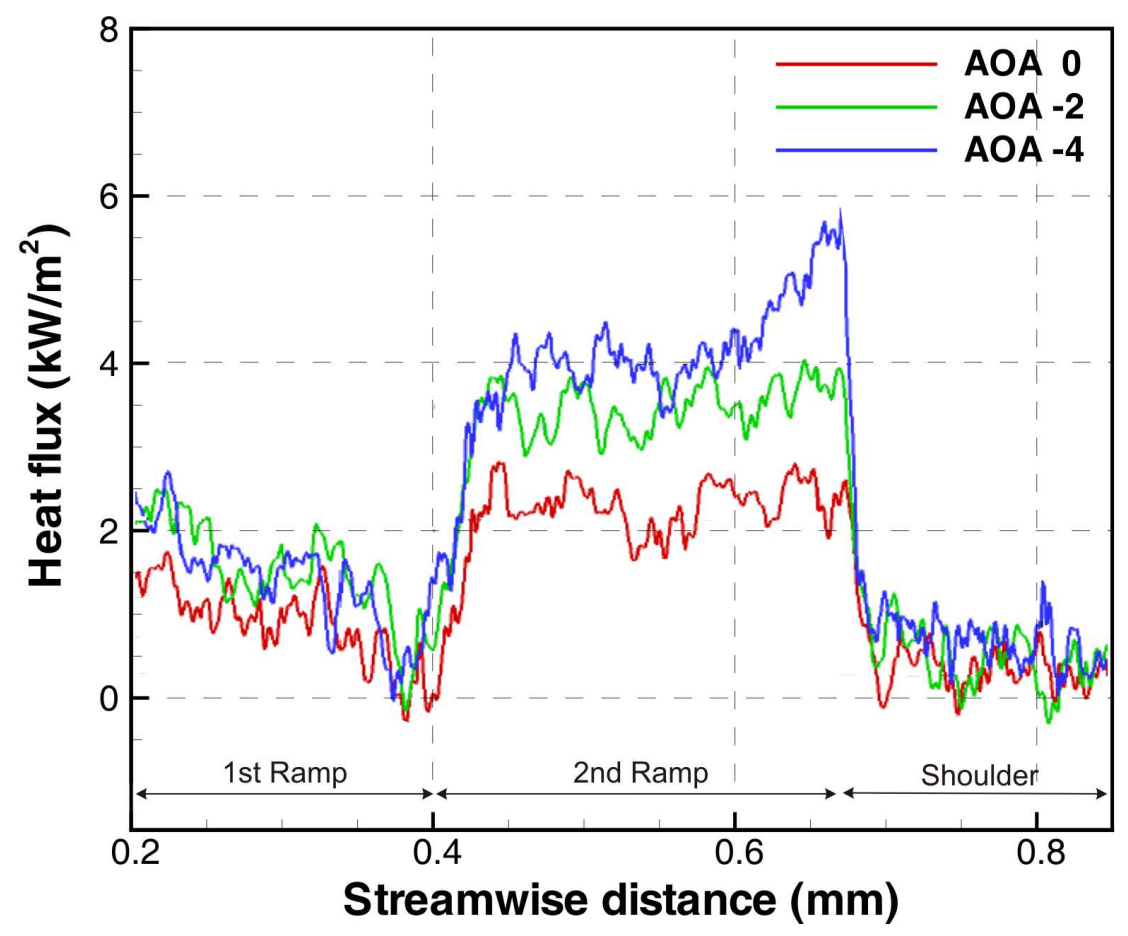

FIG. 11: Variation of heat flux along the model centreline at different incidences. 


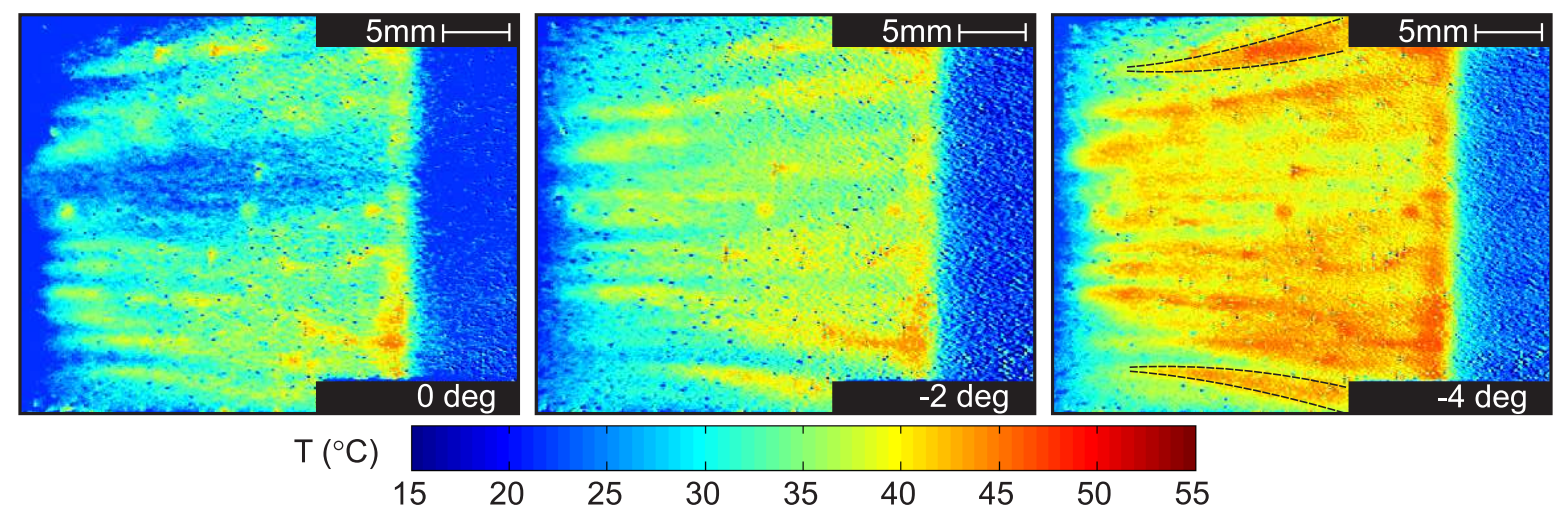

FIG. 12: TSP of the Görtler vortices formed on the second ramp at different incidences.

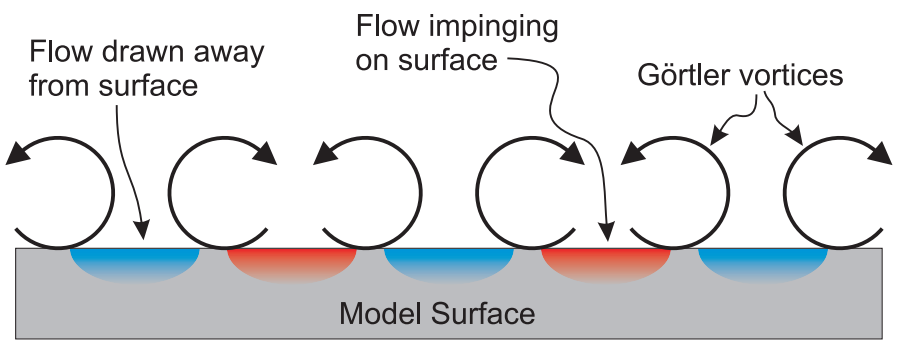

FIG. 13: Flow pattern induced by the Görtler vortices adjacent to the model surface. ${ }^{15}$ 


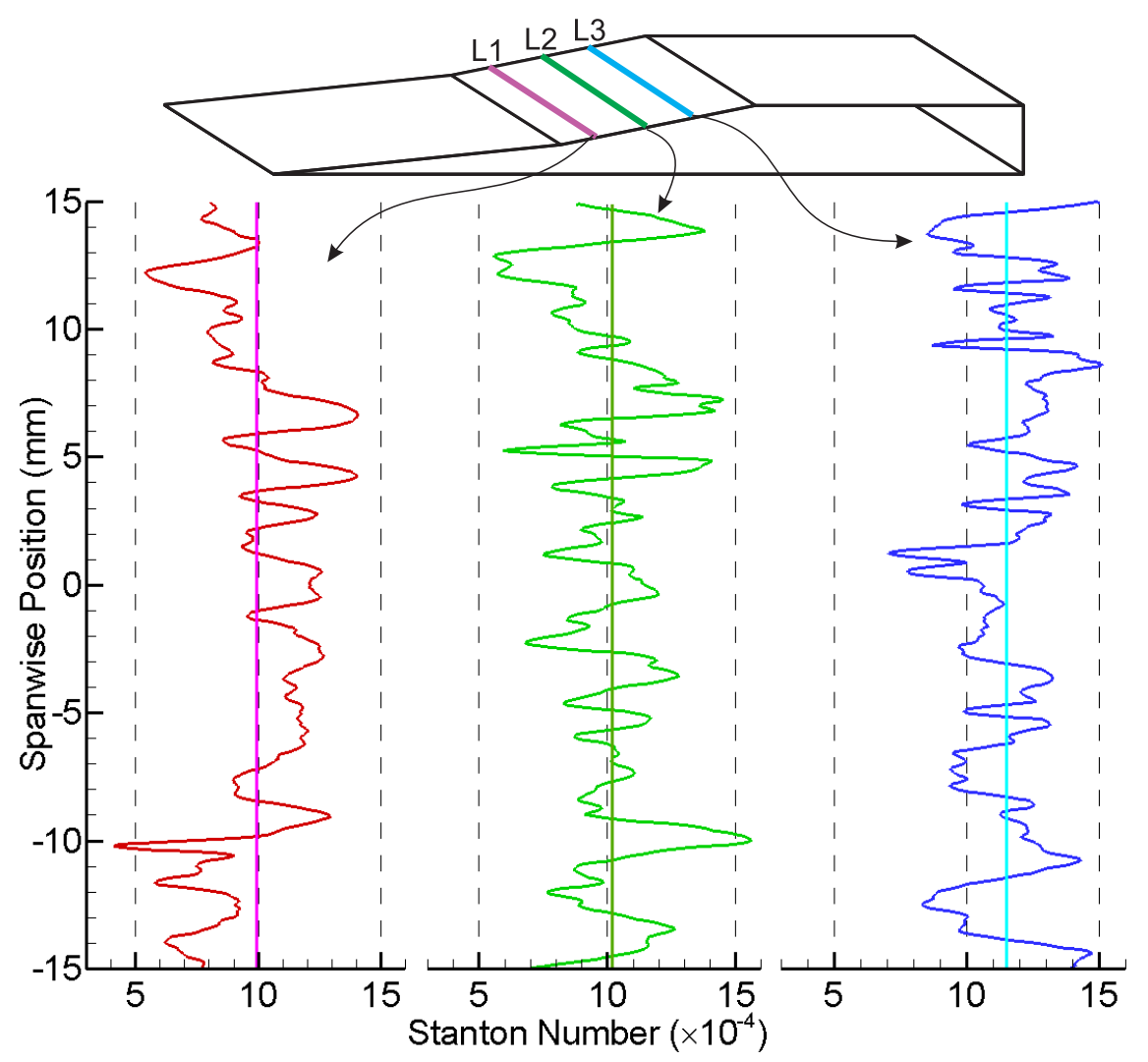

FIG. 14: Spanwise variation of Stanton number at different locations for an incidence of -4 degrees. 